\title{
Soft or hard ionization of molecules in helium nanodroplets? An electron impact investigation of alcohols and ethers
}

\author{
Shengfu Yang, Scott M. Brereton, Martyn D. Wheeler and Andrew M. Ellis* \\ Department of Chemistry, University of Leicester, University Road, Leicester, UK LE1 7 RH. \\ E-mail: andrew.ellis@le.ac.uk; Fax:+44 (0)116 2523789; Tel: +44 (0)116 2522138
}

Received 15th August 2005, Accepted 27th September 2005

First published as an Advance Article on the web 10th October 2005

Electron impact $(70 \mathrm{eV})$ mass spectra of a series of $\mathrm{C}_{1}-\mathrm{C}_{6}$ alcohols encased in large superfluid liquid helium nanodroplets ( $\sim 60000$ helium atoms) have been recorded. The presence of helium alters the fragmentation patterns when compared with the gas phase, with some ion product channels being more strongly affected than others, most notably cleavage of the $\mathrm{C}_{\alpha}-\mathrm{H}$ bond in the parent ion to form the corresponding oxonium ion. Parent ion intensities are also enhanced by the helium, but only for the two cyclic alcohols studied, cyclopentanol and cyclohexanol, is this effect large enough to transform the parent ion from a minor product (in the gas phase) into the most abundant ion in the helium droplet experiments. To demonstrate that these findings are not unique to alcohols, we have also investigated several ethers. The results obtained for both alcohols and ethers are difficult to explain solely by rapid cooling of the excited parent ions by the surrounding superfluid helium, although this undoubtedly takes place. A second factor also seems to be involved, a cage effect which favors hydrogen atom loss over other fragmentation channels. The set of molecules explored in this work suggest that electron impact ionization of doped helium nanodroplets does not provide a sufficiently large softening effect to be useful in analytical mass spectrometry.

\section{Introduction}

Fragmentation of molecular ions is commonplace in electron impact (EI) mass spectrometry. It has advantages in analysis, since it allows identification of specific functional groups through the appearance of characteristic fragment ions. However, it can also be a serious disadvantage, particularly for large molecules and for mixtures of molecules, where extensive fragmentation frequently obscures analyte assignments. As a result, alternative techniques have been developed for ionizing molecules in mass spectrometry, such as chemical ionization, matrix-assisted laser desorption ionization (MALDI) and electrospray. These techniques tend to produce ions with much less fragmentation than electron impact ionization and are often referred to as 'soft' ionization methods.

A softer form of electron impact ionization is potentially possible by attaching the analyte molecule to, or embedding the molecule within, a weakly bound cluster in the gas phase. When this cluster is ionized by EI, additional bodies are present to help absorb and remove any excess energy and if sufficiently effective this might reduce fragmentation of the analyte ion. Stace and co-workers have explored this idea using argon clusters, which were formed in a supersonic gas expansion. Several small organic molecules, such as dimethyl ether, ${ }^{1,2}$ diethyl ether, ${ }^{3}$ acetone, ${ }^{4}$ methanol ${ }^{5}$ and methyl formate, ${ }^{6}$ were added to the argon clusters and subjected to electron impact ionization. It was found that extensive fragmentation occurs even with the molecules attached to argon clusters of various sizes, although the fragment ion distributions did change from those of the isolated molecule mass spectra. The data obtained were rationalized as follows. The evidence points to a molecule sitting on the surface of the argon cluster, rather than being embedded inside. For clusters of significant size, the most likely ionization site on being struck by an electron will be an argon atom. The argon ion formed then transfers its charge to the organic molecule and simultaneously deposits several $\mathrm{eV}$ of excess energy into the organic cation (from the difference in ionization energies of the argon atom and the organic molecule). Although the surrounding argon is capable of removing some of this excess energy from the parent ion, e.g. by evaporative loss of argon atoms, for many ion channels this process is too slow to be significant and so fragmentation of the organic ion is still observed and the ionization process cannot be described as soft.

Helium nanodroplets have emerged recently as a potentially promising environment for soft electron impact ionization. The properties of helium nanodroplets are remarkable. ${ }^{7,8}$ They are droplets of liquid helium formed in supersonic gas expansions and can be composed of anything from a few dozen helium atoms to in excess of $10^{8}$ helium atoms. ${ }^{9}$ A temperature of $0.38 \mathrm{~K}$ (for ${ }^{4} \mathrm{He}$ ) is maintained through evaporative loss and any molecule colliding with the droplet is likely to stick to, and then embed itself within, the droplet. ${ }^{4} \mathrm{He}$ nanodroplets are superfluid, allowing excellent mobility of molecules within the droplets and this fact, coupled with the potentially enormous cooling rate possible by evaporative loss of the very weakly bound helium atoms, leads to the possibility of rapid cooling of parent ions formed by electron impact ionization.

A mechanism for the ionization process in helium droplets has been described by Toennies and co-workers ${ }^{10,11}$ and developed further by Janda et al. ${ }^{12,13}$ The starting point is to recognize that it is highly unlikely that an incoming electron will directly ionize a molecule deep in the interior of a helium droplet. Instead the process is thought to begin by impact of the electron with a helium atom at or near the surface of the droplet, forming $\mathrm{He}^{+}$. The positive charge can transfer between adjacent helium atoms but after several such hops it becomes localized either by ionizing the dopant molecule (if encountered in the hopping chain), or by formation of $\mathrm{He}_{2}{ }^{+}$. In either case heat is released and this can be partly or fully removed by loss of helium atoms or ions, including cluster ions such as $\mathrm{He}_{2}{ }^{+}$.

Surprisingly, there have been relatively few mass spectral studies of molecules in helium droplets. The first hint of soft ionization of dopant molecules in helium droplets came from a 
study of $\mathrm{SF}_{6}$ by the Toennies group. ${ }^{10}$ Electron impact ionization was reported to produce copious amounts of the parent ion, an ion not seen at all in conventional EI mass spectra of bare $\mathrm{SF}_{6}$. It was subsequently found that the $\mathrm{SF}_{6}{ }^{+}$signal was due to $\mathrm{SF}_{5}{ }^{+}$clustered with a water molecule, ${ }^{14}$ the distinction between $\mathrm{SF}_{6}{ }^{+}$and $\mathrm{SF}_{5}{ }^{+}-\mathrm{H}_{2} \mathrm{O}$ not being made in the original study because the mass resolution was relatively low. However, later studies have indicated that EI of doped helium droplets can be a significantly softer process than EI of bare molecules. Janda and co-workers have recorded EI mass spectra of both pure and doped helium droplets. ${ }^{12,13,15,16}$ Amongst this body of work was a report of soft ionization of NO dimers, the parent ion of which is almost entirely fragmented in the gas phase but which remains largely intact when ionized in helium droplets composed of $\sim 15000$ helium atoms. ${ }^{12}$ Most recently of all, Miller and co-workers have carried out a detailed investigation of the electron impact ionization of triphenylmethanol in helium droplets of various sizes. ${ }^{17}$ A substantial change in the fragmentation pattern was found for triphenylmethanol in helium droplets compared with EI of the isolated molecule, with the 'softening' effect increasing as the size of the helium droplet increased. Miller and co-workers also briefly presented the mass spectrum of $n$-butanol in helium droplets, where the softening effect of the helium is much smaller than seen in triphenylmethanol. This suggests that a helium droplet is not a universal 'softener' of electron impact ionization processes.

In this paper we present the first detailed study of electron impact mass spectra of a series of molecules in helium nanodroplets. The focus is mainly on alcohols, which tend to show extensive fragmentation in their gas phase EI mass spectra. However, a small number of ethers are also included for comparative purposes. By choosing a series of related molecules, our aim is to try and identify any general trends in ion fragmentation behavior in helium nanodroplets. We employ large nanodroplets with an average size of 50 000-60 000 helium atoms. Droplets of this size have the capability to dissipate $\sim 30-35 \mathrm{eV}$ of energy by evaporative loss of helium atoms, and yet we find in most cases that the helium droplet has only a modest effect on the fragmentation patterns of the molecular ions. The major exceptions to this are the two cyclic alcohols studied, cyclopentanol and cyclohexanol, where the difference between the gas phase and helium droplet spectra is striking. However, for all the molecules we find a major increase in the branching ratio for the parent ion decomposition channel involving loss of one $\mathrm{H}$ atom, which we interpret as evidence for a cage effect by the surrounding helium solvent.

\section{Experimental}

Helium droplets were produced using a pulsed nozzle. The only previous published report of helium droplets formed in a pulsed source was by Vilesov and co-workers. ${ }^{18}$ Our pulsed nozzle is similar to that employed by the Vilesov group and consists of a commercial pulsed valve (General Valve series 99) which was modified to allow operation at temperatures as low as $6 \mathrm{~K}$. The nozzle was cooled by contact with a closed-cycle cryostat (Sumitomo Industries RDK-408D cold head with CSW-71D compressor) and the temperature was measured using a diode sensor located very close to the nozzle.

The three interconnected vacuum chambers in our experimental apparatus are illustrated in the schematic diagram in Fig. 1. The pulsed nozzle and cold head are located in the first chamber, which is pumped by a diffusion pump. The helium droplet expansion produced from the nozzle is skimmed to form a droplet beam and then flows through a small pick-up cell (length $7 \mathrm{~cm}$ ) located in a second vacuum chamber. This is the point in the apparatus where analyte gases are added, the samples in this work being derived from the headspace above the corresponding liquid. The headspace vapor is carried into

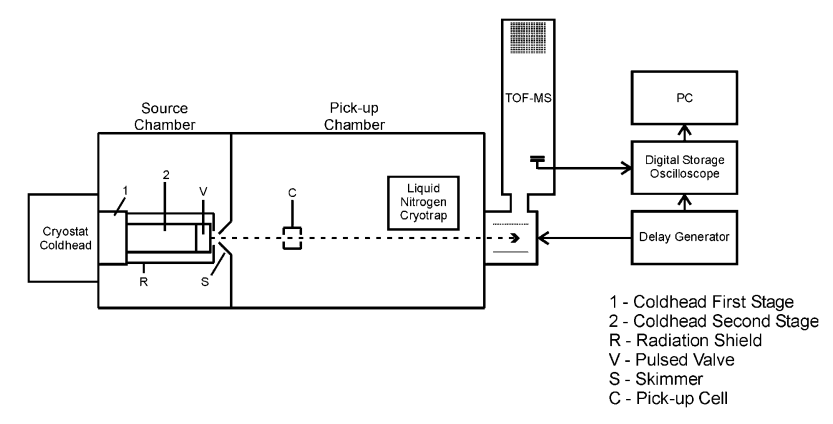

Fig. 1 Diagram showing the main components of the experimental apparatus (not to scale).

the pickup cell using helium as a carrier gas and the pressure in the pickup cell is fine tuned using two needle valves in series. The pressure is monitored by an ion gauge located on a tube on the opposite side of the pickup cell from the gas inlet tube. Typical partial pressures of the dopant gas in the pickup cell were in the region of $10^{-6}$ mbar.

The final vacuum chamber houses a reflectron time-of-flight mass spectrometer, which was designed and built by Kore Technology (Ely, UK). The mass spectrometer has a nominal resolution $(m / \Delta m)$ of 3000 and can therefore easily distinguish between ions differing in mass by one dalton. Droplets entering the source region of the mass spectrometer are ionized by a pulsed electron beam at a fixed energy of $70 \mathrm{eV}$. The firing of the electron beam is timed to coincide with the arrival of each pulse of helium droplets. The mass spectrometer is equipped with a dual microchannel plate detector, a preamplifier, and an ion counting system for accumulating the mass spectral data.

In the experiments reported here the nozzle was set for a nominal opening time of $150 \mu$ s and fired at a repetition rate of $10 \mathrm{~Hz}$. High purity helium (99.9999\%) was employed at a typical stagnation pressure of 20 bar and a nozzle temperature of $11 \mathrm{~K}$. Under similar experimental conditions Vilesov and coworkers determined an average droplet size of $5 \times 10^{4}$ helium atoms. ${ }^{18}$ We have independently determined the droplet sizes in our experiments using a procedure that has been described in detail elsewhere and parallels that used by many other research groups. ${ }^{19}$ Briefly, the probability that a droplet acquires one or more dopant molecules is governed by Poisson statistics. Consequently, the observed variation in monomer or cluster ion signals with partial pressure can be fitted to a Poisson distribution by using the droplet size as the fitting parameter. By this means we obtained a value of $\sim 6 \times 10^{4}$ helium atoms, which is in good agreement with Vilesov's determination.

Since this work focuses on the electron impact ionization of single dopant molecules in helium droplets, the partial pressure of the dopant gas in the pick-up cell was kept deliberately low in order to avoid the formation of significant quantities of molecular clusters, whose presence might complicate the interpretation of mass spectral fragmentation patterns. In experiments to be reported elsewhere, we have also carried out measurements at much higher dopant partial pressures to probe the fragmentation of alcohol cluster ions in helium nanodroplets. For most of the alcohols studied here the cluster mass spectra are dominated by protonated cations, $\mathrm{M}_{n} \mathrm{H}^{+}$, where $\mathrm{M}$ is an alcohol monomer and $\mathrm{M}_{n}$ is the corresponding $n$-mer cluster. These ions must originate from larger clusters, most likely the fragmentation of $\mathrm{M}_{n+1}{ }^{+}$ions. Consequently, the integrated ratio of monomer ion signals to the $\mathrm{MH}^{+}$signal, which is seen in all of our mass spectra, can be used to estimate the ratio of monomer to dimer concentrations. In all instances this ratio was found to be $\gg 10$, showing that the dimers and larger clusters play a negligible role in the work reported here.

All samples were obtained from standard chemical suppliers with a minimum purity of $97 \%$, and in most cases better than $99 \%$. Each sample was placed in a small cylindrical stainless 
steel container and degassed thoroughly prior to use. Despite attempts to minimize background gases, substantial signals from $\mathrm{N}_{2}, \mathrm{O}_{2}$, and $\mathrm{H}_{2} \mathrm{O}$ were seen in all experiments. This reflects the weakness of the helium droplet ion signals, rather than excessive amounts of background gases, and typically several thousand spectra were accumulated to attain an adequate signal-to-noise ratio. The background gases originated mainly from within the mass spectrometer chamber (which nevertheless gave a base pressure $<10^{-8}$ mbar) and the amounts of these contaminants picked up by the helium droplets were found to be negligible.

\section{Results}

The molecules chosen for investigation were mainly alcohols, although a few ethers were also included. The precise choice was determined partly by availability of the compounds in our laboratory and also by the need to avoid those compounds that gave too many fragments coinciding with $\mathrm{He}_{n}{ }^{+}$cluster ions (as well as other abundant background ions such as $\mathrm{H}_{2} \mathrm{O}^{+}, \mathrm{N}_{2}{ }^{+}$ and $\mathrm{O}_{2}{ }^{+}$). The helium droplet spectra were restricted to electron impact energies of $70 \mathrm{eV}$, since this is the typical impact energy employed in analytical EI mass spectrometry. For comparison with the helium droplet data, mass spectra of all the chosen molecules were also recorded with the same instrument but in the absence of helium droplets. This gave mass spectra of the bare molecules and these were found to be very similar to those available in the NIST mass spectral database. $^{20}$

\subsection{Alcohols}

Primary alcohols. It is well known that the fragmentation patterns of primary alcohols become steadily more complex as the length of the hydrocarbon chain(s) increase. ${ }^{21,22}$ The parent ion is always a minor product at $70 \mathrm{eV}$ in the gas phase and for the longer chain alcohols is barely detectable. Ionization of saturated alcohols is initiated by loss of an electron from the oxygen atom. In the case of the lighter alcohols the fragmentation that then results is dominated by the formation of oxonium ions, as shown for ethanol below:

$$
\mathrm{CH}_{3} \mathrm{CH}_{2} \mathrm{OH} \rightarrow \mathrm{CH}_{3} \mathrm{CH}_{2} \mathrm{O}^{+} \mathrm{H} \rightarrow \mathrm{CH}_{3}+\mathrm{CH}_{2}=\mathrm{O}^{+} \mathrm{H}
$$

For ethanol the cleavage of the $\mathrm{C}-\mathrm{C}$ bond at the $\alpha$ carbon leads to the characteristic oxonium fragment ion at $m / z=31$, which is the major ion in the $70 \mathrm{eV}$ EI mass spectrum in the gas phase (see the lower panel in Fig. 2). However, cleavage of the $\mathrm{C}-\mathrm{H}$ bond at the $\alpha$ carbon is also possible leading to the oxonium

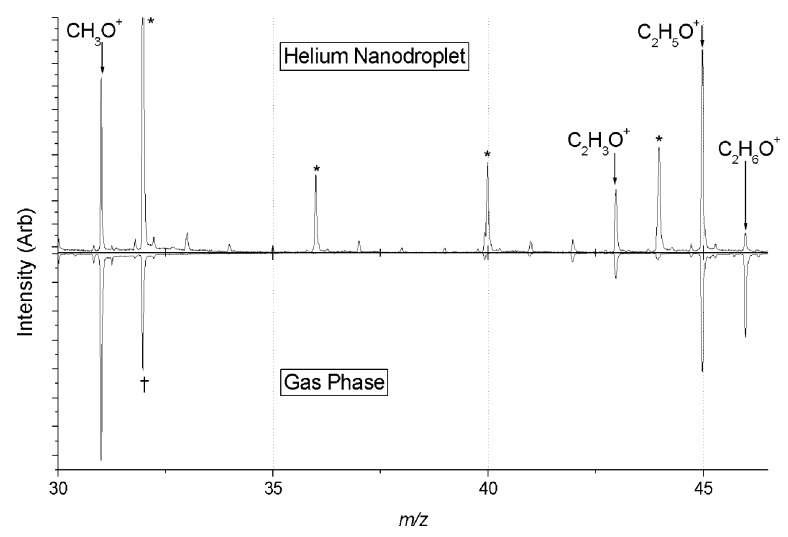

Fig. 2 Mass spectra from ethanol in helium nanodroplets and in the gas phase at room temperature. The peaks marked with asterisks in the helium droplet spectrum correspond to helium cluster ion peaks. The peak marked $\dagger$ in the lower spectrum is from residual $\mathrm{O}_{2}{ }^{+}$in the vacuum system, and which of course will also contribute to the peak at the same position in the upper spectrum. ion $\mathrm{CH}_{3} \mathrm{C}(\mathrm{H})=\mathrm{O}^{+} \mathrm{H}$ at $m / z=45$, one mass unit below the parent ion.

The upper panel in Fig. 2 shows the EI mass spectrum of ethanol in helium nanodroplets. In addition to peaks arising from the ionization of ethanol, the spectrum shows a series of intense $\mathrm{He}_{n}{ }^{+}$cluster peaks characteristic of the electron impact ionization of helium nanodroplets. Although it is in principle possible to subtract the $\mathrm{He}_{n}{ }^{+}$peaks from the spectrum to reveal the organic ion peaks only, by recording a second spectrum with the ethanol gas source turned off, in practice pulse-to-pulse fluctuations in gas intensity lead to a very noisy difference spectrum. We have therefore chosen to present raw mass spectra in this work. For each molecule we have carefully checked that the contribution to the mass spectrum from molecules not picked up by the helium droplets was negligible. This was achieved by raising the temperature of the nozzle to destroy the droplets, leaving all other experimental conditions the same. It is also noteworthy that all the helium nanodroplet mass spectra presented in this paper show total $\mathrm{He}_{n}{ }^{+}$signals far in excess of the organic ion signals. This is consistent with the expected large size of the helium nanodroplets quoted earlier, for which a low charge transfer probability to the organic dopant molecule is expected.

There are substantial differences in relative intensities of the mass peaks arising from ethanol in helium nanodroplets compared with the gas phase spectrum. The two oxonium ions remain the most abundant organic ions, but the peak due to loss of the $\alpha$ hydrogen is now stronger than that due to cleavage of the methyl group. The parent ion is relatively weak in the helium droplet spectrum but the peak at $m / z=43$, which corresponds to loss of $\mathrm{H}_{2}$ from the $\mathrm{CH}_{3} \mathrm{C}(\mathrm{H})=\mathrm{O}^{+} \mathrm{H}$ oxonium ion, has gained intensity relative to that of the parent ion and is now much the stronger of the two peaks. Clearly the helium droplet has a substantial effect on the relative intensities of the peaks, although the mass spectrum is still recognizably that of ethanol.

The other primary alcohols investigated in this work were 2methyl-1-propanol, $n$-butanol, and 1-pentanol. For these molecules fragmentation channels other than just oxonium ion formation become significant. For 2-methyl-1-propanol and $n$-butanol there are differences in fragmentation patterns between the gas phase and helium droplet spectra, but as for ethanol these differences for the majority of ions are not profound. The main exception is the channel due to loss of the $\alpha$ hydrogen atom to give a peak at $M-1$, where $M$ is $m / z$ for the parent ion. The $M-1$ peaks for both molecules are dramatically enhanced relative to the gas phase spectrum, in line with the finding for ethanol. The parent ion peaks also show much higher intensities than in the gas phase spectra, although they remain weak. This is particularly noteworthy for $n$-butanol, where in the gas phase the parent ion is barely observable. The mass spectra recorded for $n$-butanol are shown in Fig. 3. As part of a detailed study concentrating mainly on triphenylmethanol, Lewis and co-workers in a recent publication briefly presented the mass spectrum of $n$-butanol in helium droplets. ${ }^{17}$ In contrast to triphenylmethanol, where the helium solvent produces softer ionization resulting in a dramatic enhancement of the parent ion peak, in $n$-butanol the parent ion was found to remain weak even in very large droplets $(4 \times$ $10^{4}$ helium atoms). Lewis et al. presented this spectrum to counter the impression that helium droplets always act as strong quenchers of ion fragmentation processes. We reach essentially the same conclusion about the low intensity of the parent ion, but the much higher mass resolution in our experiments clearly shows the dramatic enhancement in the $M-1$ channel.

The most marked differences between gas phase and helium droplet spectra for the primary alcohols investigated were found for 1-pentanol. The parent ion is several hundred times weaker than the prominent fragment ions in the gas phase 


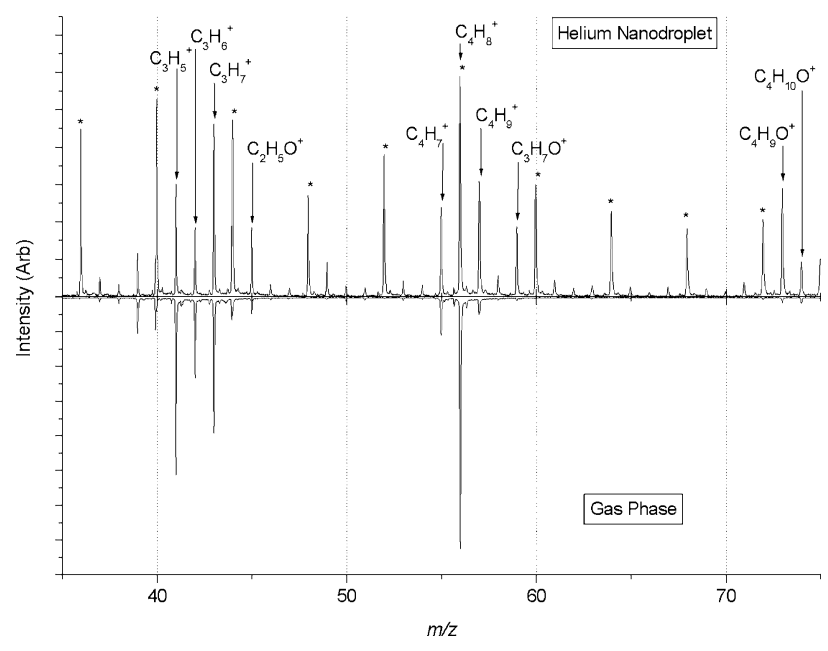

Fig. 3 Mass spectra from $n$-butanol in helium nanodroplets and in the gas phase at room temperature. The peaks marked with asterisks in the helium droplet spectrum correspond to helium cluster ion peaks.

spectrum, but there is evidence from the helium droplet spectrum (see Fig. 4) that it gains considerably in intensity (the parent ion peak coincides with the $\mathrm{He}_{22}{ }^{+}$peak but there is a clear enhancement in intensity relative to the surrounding helium cluster peaks which suggests a contribution from the parent ion). The $M-1$ peak, which is also barely detectable in the gas phase spectrum, is comparable in intensity to all the major fragment peaks in the helium droplet spectrum. However, there are other striking changes in relative intensities in moving from the gas phase to helium droplets. For example, the major peak in the gas phase spectrum is at $m / z=42$, which corresponds to the loss of both $\mathrm{H}_{2} \mathrm{O}$ and $\mathrm{C}_{2} \mathrm{H}_{4}$. It has been proposed that the loss of these molecules occurs simultaneously through a cyclic transition state, ${ }^{21}$ as illustrated mechanistically below.

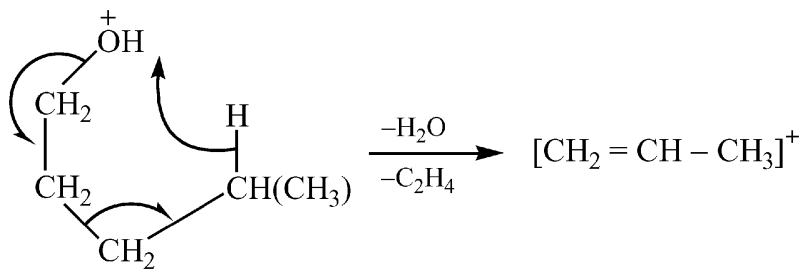

The fragment ion arising from this process suffers a major decline in relative abundance in the helium droplet mass

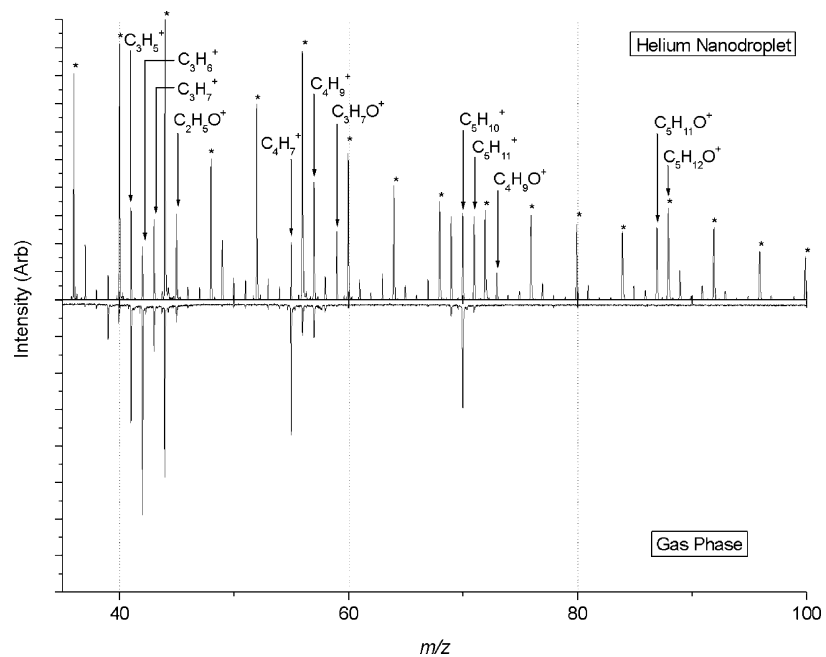

Fig. 4 Mass spectra from 1-pentanol in helium nanodroplets and in the gas phase at room temperature. The peaks marked with asterisks in the helium droplet spectrum correspond to helium cluster ion peaks.

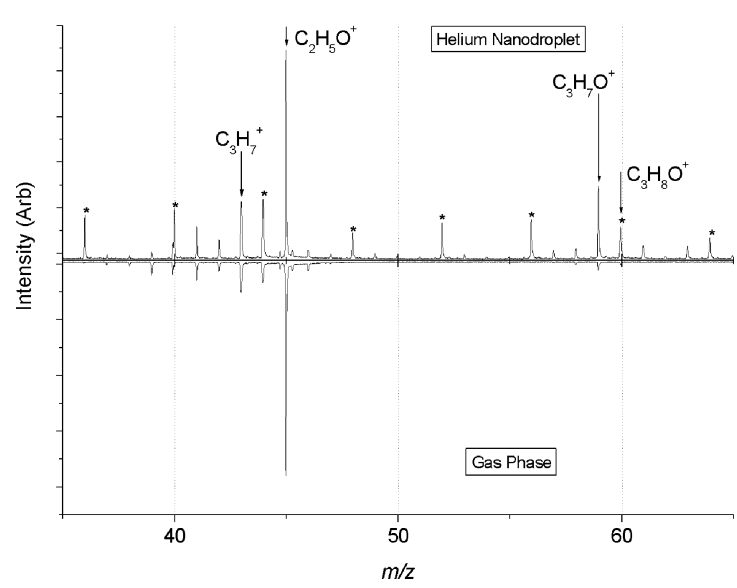

Fig. 5 Mass spectra from 2-propanol in helium nanodroplets and in the gas phase at room temperature. The peaks marked with asterisks in the helium droplet spectrum correspond to helium cluster ion peaks.

spectrum, while some other nearby peaks gain in their relative intensities.

Secondary and tertiary alcohols. Mass spectra of four secondary alcohols (2-propanol, 2-butanol, 2-pentanol and 2methyl-3-pentanol were studied along with one tertiary alcohol, $t$-butanol. The findings were similar to those reported for the primary alcohols, and as an illustration we show the gas phase and helium nanodroplet EI mass spectra of 2-propanol in Fig. 5. Both the parent ion $(M)$ and the $M-1$ peaks are minor features in the gas phase spectrum, the major peak being due to the oxonium fragment $\mathrm{CH}_{3} \mathrm{C}(\mathrm{H})=\mathrm{O}^{+} \mathrm{H}$ at $m / z=45$. The helium droplet enhances the proportion of the $M-1$ ion, but that aside there are strong similarities between the gas phase and helium droplet spectra.

Cyclic alcohols. Two cyclic alcohols were selected for investigation, cyclopentanol and cyclohexanol. It is for these two molecules that the helium solvent shows most impact. Fig. 6 shows mass spectra recorded for cyclopentanol. The most notable observation is that the weak parent ion peak in the gas phase spectrum is transformed into the most intense organic ion signal in the helium droplet spectrum. Almost as abundant as the parent ion is the $M-1$ ion, a species absent from the gas phase spectrum. All the fragmentation channels active in the gas phase spectrum are also seen in the helium droplet spectrum, but there are clear differences in branching ratios. The major peak in the $70 \mathrm{eV}$ gas phase spectrum of cyclopentanol is at $m / z=57$, which corresponds to the

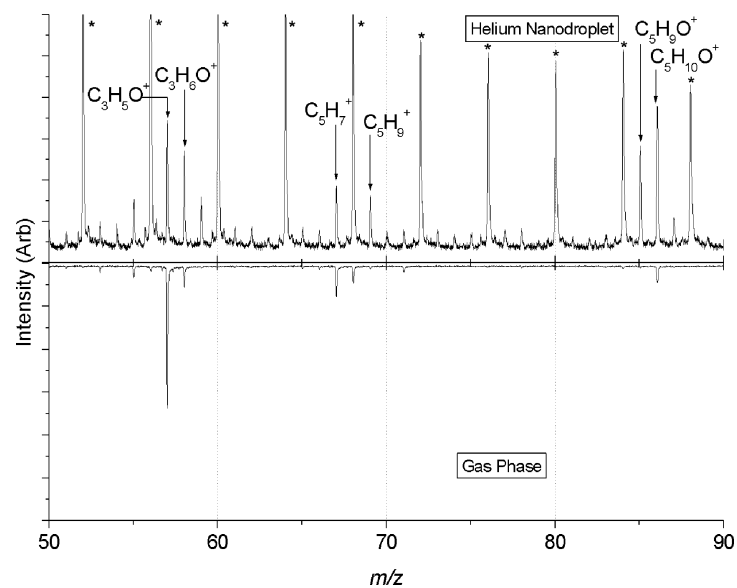

Fig. 6 Mass spectra from cyclopentanol in helium nanodroplets and in the gas phase at room temperature. The peaks marked with asterisks in the helium droplet spectrum correspond to helium cluster ion peaks. 
$\mathrm{CH}_{2}=\mathrm{CH}-\mathrm{CH}=\mathrm{O}^{+} \mathrm{H}$ ion formed by a ring-opening process after initial ionization at the oxygen atom. ${ }^{21}$ This is still a major peak in the helium droplet spectrum, but the importance of this channel in helium has been reduced relative to many of the other fragmentation channels. The key findings for cyclohexanol are similar to those for cyclopentanol.

\subsection{Ethers}

Diethyl ether, diisopropyl ether and methyl $t$-butyl ether were investigated. In many respects the mass spectrum of diethyl ether in helium nanodroplets resembles that seen for the isolated molecule in the gas phase. Paralleling the alcohols, oxonium ion formation can occur by $\alpha$-cleavage, ${ }^{21}$ as shown for the diethyl ether cation below:

$$
\begin{aligned}
\mathrm{CH}_{3} \mathrm{CH}_{2}-\mathrm{O}^{+}-\mathrm{CH}_{2} \mathrm{CH}_{3} & \rightarrow \mathrm{CH}_{3} \mathrm{CH}_{2} \mathrm{O}^{+}=\mathrm{CHCH}_{3}+\mathrm{H} \\
& \rightarrow \mathrm{CH}_{3} \mathrm{CH}_{2} \mathrm{O}^{+}=\mathrm{CH}_{2}+\mathrm{CH}_{3}
\end{aligned}
$$

The processes above yield fragment ions at $m / z=73$ and 59 in addition to the parent ion peak at 74 . The $M-1$ peak at 73 is very weak in the gas phase spectrum but the ion at $m / z=59$ is one of the more abundant fragments. Secondary fragmentation is a characteristic feature in the mass spectra of ethers, some of the main channels for diethyl ether being

$$
\begin{aligned}
\mathrm{CH}_{3} \mathrm{CH}_{2} \mathrm{OCH}_{2}^{+} & \rightarrow \mathrm{C}_{3} \mathrm{H}_{5}^{+}+\mathrm{H}_{2} \mathrm{O} \\
& \rightarrow \mathrm{CH}_{2} \mathrm{OH}^{+}+\mathrm{C}_{2} \mathrm{H}_{4} \\
& \rightarrow \mathrm{C}_{2} \mathrm{H}_{5}^{+}+\mathrm{CH}_{2} \mathrm{O}
\end{aligned}
$$

Gas phase and helium droplet mass spectra of diethyl ether are compared in Fig. 7. They are similar in many respects, although there are some differences in relative intensities. However, as per the alcohols, the one major difference is a dramatic enhancement of the $M-1$ channel.

Diisopropyl ether is an interesting target for EI mass spectrometry in helium droplets because in the corresponding gas phase spectrum the parent ion has a low abundance. Instead there are a number of different fragment ions, with by far the most abundant being that at $m / z=45$. A plausible fragmentation scheme that accounts for the major peaks is as follows:

$$
\begin{aligned}
& \left(\mathrm{CH}_{3}\right)_{2} \mathrm{CH}-\mathrm{O}^{+}-\mathrm{CH}\left(\mathrm{CH}_{3}\right)_{2}
\end{aligned}
$$

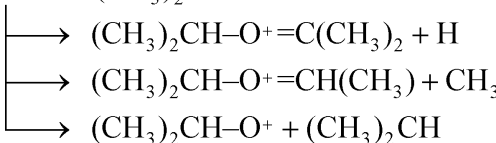
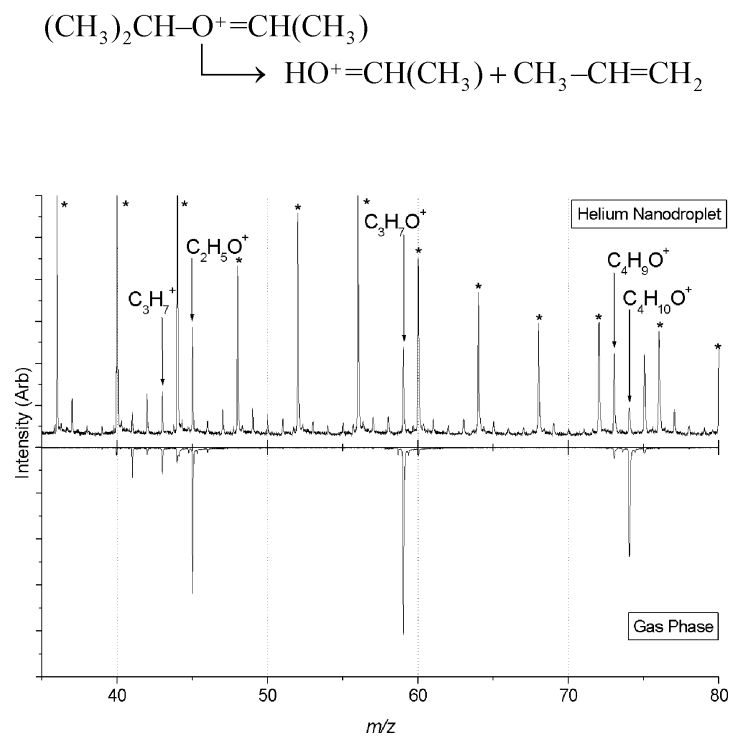

Fig. 7 Mass spectra from diethyl ether in helium nanodroplets and in the gas phase at room temperature. The peaks marked with asterisks in the helium droplet spectrum correspond to helium cluster ion peaks.

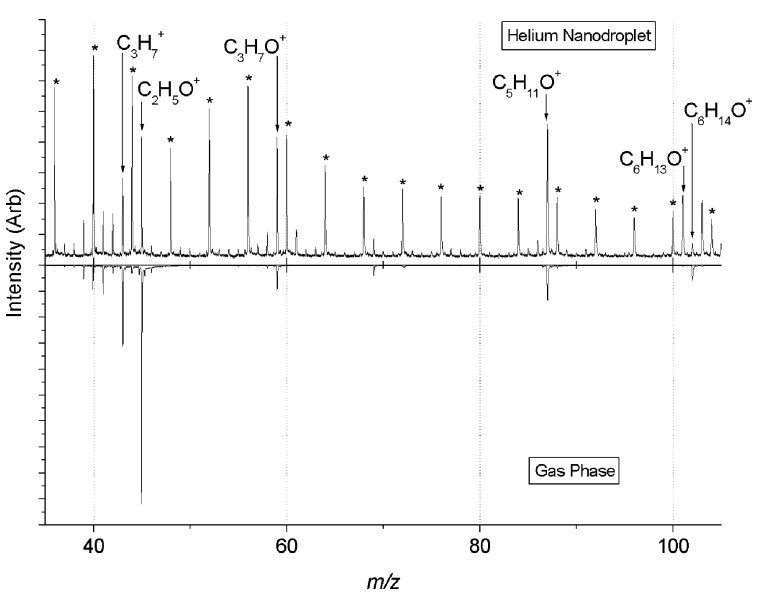

Fig. 8 Mass spectra from diisopropyl ether in helium nanodroplets and in the gas phase at room temperature. The peaks marked with asterisks in the helium droplet spectrum correspond to helium cluster ion peaks.

The helium droplet spectrum differs from the isolated molecule spectrum in several ways (see Fig. 8). As seen for other molecules, a relatively strong $M-1$ peak at $m / z=101$ is observed which has negligible intensity in the gas phase spectrum. Particularly striking in Fig. 8 is the dramatic reduction in the abundance of the $\mathrm{HO}^{+}=\mathrm{CH}\left(\mathrm{CH}_{3}\right)$ cations at $m / z=45$ relative to the other organic peaks. A peak at 59, corresponding to the isopropoxy cation, $\left(\mathrm{CH}_{3}\right)_{2} \mathrm{CH}-\mathrm{O}^{+}$, is now equally abundant and yet in the gas phase this is a very minor product. The effect of the helium on the mass spectrum is clearly more pronounced for diisopropyl ether than for diethyl ether.

Finally, we recorded the EI mass spectra of methyl $t$-butyl ether. For this example the helium droplets had a much smaller effect on the mass spectra than seen for diisopropyl ether, the only notable difference being, once again, an enhancement in the $\mathrm{M}-1$ channel.

\section{Discussion}

The above results clearly show that electron impact ionization of helium droplets doped with alcohols and ethers gives different ion fragmentation patterns when compared with gas phase samples. However, it is also clear that in most cases these differences are quantitative rather than qualitative and vary in extent from molecule to molecule. The question arises as to what role the helium plays in the ionization and subsequent fragmentation process, and therefore what is the source of the differences between ionization in a helium droplet and in the gas phase?

An important distinction between gas phase EI and helium droplet EI concerns the energetics. As discussed in the Introduction, an incoming electron is unlikely to strike a dopant molecule in a helium droplet unless it is at or very near the surface. We would expect all the molecules chosen for investigation in this work to be fully solvated by the helium, i.e. on average they will reside within the droplet. As a result, the initial ionization event will in almost every case involve production of $\mathrm{He}^{+}$, which can then subsequently transfer its charge to the dopant molecule. The first ionization energy of a helium atom is $24.6 \mathrm{eV}$, with a similar value being expected in a helium droplet. Typical first ionization energies of simple alcohols fall in the range of $10-11 \mathrm{eV}$, with those of ethers being marginally lower. Consequently, a maximum of 15-16 $\mathrm{eV}$ of excess energy could be deposited into the parent ion on charge transfer from $\mathrm{He}^{+}$. This is clearly a substantial amount of energy, although much less than that potentially available from $70 \mathrm{eV}$ electron impact on the bare molecule. Could this difference in available energy account for all of the differences between the gas phase and helium droplet spectra? We can 


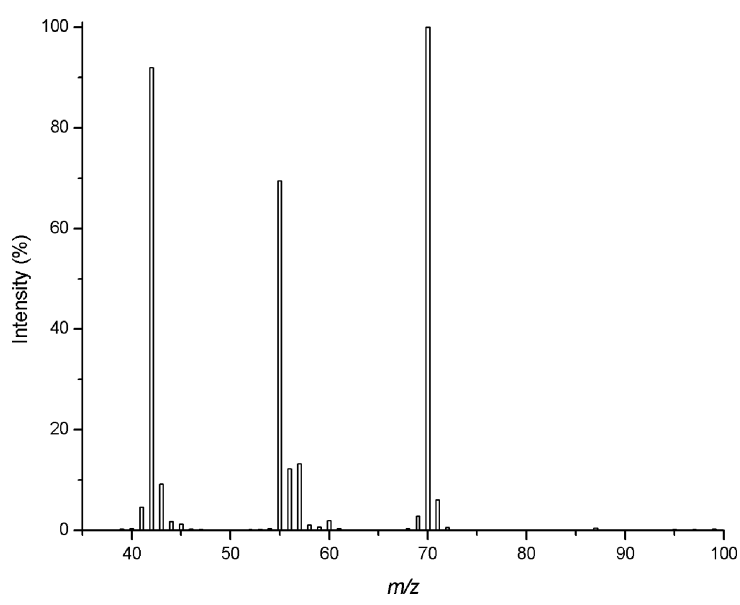

Fig. 9 Mass spectrum of gaseous 1-pentanol at an electron impact energy of $15 \mathrm{eV}$. This spectrum was recorded on a Kratos MS 890 magnetic sector mass spectrometer and focuses on the same mass region as that shown in Fig. 4.

easily show that this is not the case by recording low energy electron impact mass spectra in the gas phase. As an illustration, Fig. 9 shows the mass spectrum of gaseous 1-pentanol at $15 \mathrm{eV}$ impact energy. This corresponds to a much lower energy $(\sim 5 \mathrm{eV})$ available for depositing into the parent ion than is the case in a helium droplet and yet the mass spectrum in Fig. 9 is very different from the helium droplet spectrum in Fig. 4. Clearly, the greater excess energy available from $70 \mathrm{eV}$ electron impact ionization of the bare organic molecules is not in itself sufficient to account for the differences between gas phase and helium droplet mass spectra.

Another factor in play for helium droplets is the potential for cooling the parent and fragment ions. If this takes place on a timescale comparable to, or faster than, the dissociation rate of the energized ion, then this could reduce the degree of fragmentation. Superfluid helium is known for its extremely high thermal conductivity, and claims have been made for an evaporative cooling rate as high as $10^{10} \mathrm{~K} \mathrm{~s}^{-1} \cdot{ }^{23}$ A droplet size of 60000 helium atoms is more than large enough to dissipate $15 \mathrm{eV}$ of excess energy through evaporative loss alone $\left(\sim 1600\right.$ helium atoms $\left.\mathrm{eV}^{-1}\right)$. In the limit of full dissipation of the excess energy we would see only parent ions, which is clearly not what is observed. However, a partial cooling effect might account for some of the changes in mass spectra between the gas phase and helium droplets.

To explore this further, we consider a specific example, ethanol. The dissociation pathways of ethanol cations and their energetics have been extensively studied. The best available threshold energies for formation of the parent ion and the two main fragments are $10.64 \mathrm{eV}\left(\mathrm{C}_{2} \mathrm{H}_{5} \mathrm{OH}^{+}\right), 10.80 \mathrm{eV}$ $\left(\mathrm{CH}_{3} \mathrm{CHOH}^{+}\right)$and $11.4 \mathrm{eV}\left(\mathrm{CH}_{2} \mathrm{OH}^{+}\right){ }^{20}$ At $70 \mathrm{eV}$ in the gas phase the formation of the oxonium ion $\mathrm{CH}_{2} \mathrm{OH}^{+}$is favored over the alternative oxonium ion $\mathrm{CH}_{3} \mathrm{CHOH}^{+}$. However, in view of their relative appearance energies at much lower excitation energies the branching ratio should begin to shift in favor of $\mathrm{CH}_{3} \mathrm{CHOH}^{+}$over $\mathrm{CH}_{2} \mathrm{OH}^{+}$. This is supported by photoionization studies, and in particular a PEPICO study by Niwa and co-workers found that the crossover point occurs at a photon energy of $\sim 11.5 \mathrm{eV}{ }^{24}$ The same group was not able to distinguish between the amounts of $\mathrm{CH}_{3} \mathrm{CHOH}^{+}$and the parent ion due to a lack of mass resolution. However, Bowen and Maccoll have recorded a gas phase mass spectrum at an electron impact energy of $12.1 \mathrm{eV}$ and found that, in addition to an excess of $\mathrm{CH}_{3} \mathrm{CHOH}^{+}$over $\mathrm{CH}_{2} \mathrm{OH}^{+}$, the parent ion is the most abundant ion. ${ }^{25}$ On the basis of these data, we can explain the preponderance of $\mathrm{CH}_{3} \mathrm{CHOH}^{+}$over $\mathrm{CH}_{2} \mathrm{OH}^{+}$in our helium droplet experiments by invoking extensive and rapid cooling of the parent ion. However, the presence of a weak parent ion, together with a substantial
$\left[M-\mathrm{H}_{3}\right]$ peak, whose threshold appearance energy has been determined as $14.5 \mathrm{eV},{ }^{20}$ suggests that rapid ion cooling is not the only factor affecting the fragmentation pattern.

The one feature common in all of the helium droplet mass spectra recorded in this work is the greatly enhanced branching ratio for hydrogen atom loss compared with the gas phase spectrum. What is often a minor, or even negligible, fragmentation channel in the gas phase becomes a major channel in every one of the molecules investigated. As already seen for the ethanol cation, the loss of a hydrogen atom from the $\alpha$ carbon atom to form an oxonium ion is a relatively low energy fragmentation channel which is available to all of the molecules studied in this work. Extensive cooling by the helium matrix is therefore more likely to favor this channel over more energetic fragmentation processes. However, the dramatic enhancement in the $M-1$ channel without, in most cases, any comparable increase in parent ion production, suggests that energetics alone does not account for the differences between the gas phase and helium droplet spectra.

A possible explanation is that the helium surrounding the initially formed cation acts to some extent as a cage, favoring the escape of small energetic fragments over slower moving heavy species. A recent study of VUV excitation of water molecules in helium nanodroplets has shown that predissociation (to form $\mathrm{OH}+\mathrm{H}$ ) from electronically excited states is not hindered by the surrounding helium, demonstrating that the cage effect for $\mathrm{H}$ atom loss is negligible. ${ }^{26}$ The predissociation of neutral molecules is not exactly analogous to the situation in this work, where we are dealing with the dissociation of cations. The helium atoms immediately adjacent to the cation will form a more tightly bound 'sheath' around the dopant species than in the neutral case, providing more of a barrier to fragmentation. For example, path integral Monte Carlo calculations on $\mathrm{Na}^{+} \mathrm{He}_{n}$ clusters show that the layer of helium immediately surrounding the ion behaves like highly compressed solid helium due to the strong ion-induced dipole forces between $\mathrm{Na}^{+}$and helium atoms. ${ }^{27}$ The larger ions studied in the present work will produce a less tightly bound inner solvent shell but we nevertheless expect this helium layer to possess a density and rigidity characteristic of solid helium. A cage effect by this solvent layer would favour hydrogen atom loss over ejection of larger fragments since the departure of the former requires less disruption of the rigid solvent shell. The occurrence of a cage effect might also account for the decline in the $\mathrm{H}_{2} \mathrm{O}+\mathrm{C}_{2} \mathrm{H}_{4}$ loss channel for the 1-pentanol cation. As mentioned earlier, this fragmentation process is likely to proceed through a cyclic transition state whose formation will be obstructed by the tightly bound helium solvation layer. However, the above comments neglect the fact that the initially formed cation is not cold, as would be the case with an atomic ion, but is highly energized as a result of the charge transfer process. Cooling of the ion by the helium droplet may heat the helium layers in close proximity to the ion to the point where formation of an effective rigid or semi-rigid solvation cage is impossible. A full interpretation is dependent on the relative rates of ion cooling by the helium versus unimolecular ion fragmentation. This is a complex dynamical problem and theoretical studies would be of great assistance in elucidating the detailed role of the helium in unimolecular ion fragmentation.

Finally, we comment on the role of the size of the helium droplets. The droplets in this work are comparable in size to the largest used in the EI mass spectrometric study of triphenyl methanol by Lewis et al. ${ }^{17}$ and are considerably larger than those employed by Janda and co-workers. ${ }^{12,13,15,16}$ It is conceivable that even larger droplets might achieve still more effective ion cooling, perhaps reaching the point at sufficiently large sizes ( $\gg 10^{5}$ helium atoms) where the parent ion is the only product. However, even if this were the case, it is not clear that such large droplets will eject only bare ions, and may 
instead yield ions partly solvated with helium atoms (as seen in the ionization of atoms in helium droplets $\left.{ }^{15,16}\right)$. In addition, the probability of charge transfer from $\mathrm{He}^{+}$to the dopant molecule diminishes rapidly as the size of the droplet increases. Lewis et al. found their maximum usable droplet size to be 40000 helium atoms, the dopant ion signal being unacceptably small for larger droplets. In our work we too obtain weak dopant ion signals and require averages of several thousand mass scans to achieve a respectable signal-to-noise ratio. It seems likely that larger droplet sizes will not be effective for EI mass spectrometry and so the conclusions reached in this work about the quenching of ion fragmentation are at the limit of what is practically achievable with helium droplets.

\section{Conclusions}

The alcohols and ethers chosen for investigation in this work serve to illustrate the key effects of the helium solvent on electron impact mass spectra and allow us to draw some general conclusions. First, the influence of the helium is not the same for all molecules. For example, the $70 \mathrm{eV}$ EI mass spectra of aliphatic alcohols in helium nanodroplets broadly resemble those in the gas phase. There are some consistent differences in relative fragment intensities, most notably the major enhancement of the $M-1$ peaks in the presence of helium. The situation differs, however, for the cyclic alcohols, where the impact of the helium is more dramatic. The $M-1$ peak is again enhanced considerably relative to other fragmentation channels, but the key difference for the two cyclic alcohols investigated here, cyclopentanol and cyclohexanol, is that the parent ions become the major ions in the mass spectrum, in contrast to the gas phase where they are very minor products. These are clear examples of a softening effect produced by the helium matrix.

Our findings indicate that rapid cooling of parent ions by the helium does take place, which in turn affects the branching ratios for ion fragmentation pathways. However, this cooling is incomplete, even for the large droplets employed in the present experiments, and cooling alone does not appear to account for all of the observations, particularly the major role played by the loss of a hydrogen atom from the parent ion in helium droplets. We tentatively suggest that a cage effect is also in operation which enables facile departure of hydrogen atoms at the expense of channels that produce larger fragments.

Finally, we note that even though we have found substantial quantitative changes in the mass spectra of several molecules in helium droplets compared with the gas phase, these changes are unlikely to be helpful to an analytical chemist who is seeking a new type of soft ionization. Of course the situation for molecular ions larger than those studied here, which generally fragment on a slower timescale than small molecules, may be more dramatic and the recent study on triphenylmethanol by Lewis et al. indicates that this may be the case. ${ }^{17}$ However, we conclude from the present study of small and medium sized alcohols and ethers, and a separate study of the EI mass spectra of haloalkanes, ${ }^{28}$ that fragmentation of parent ions remains a major process in the electron impact ionization of molecule-doped helium nanodroplets.

\section{Acknowledgements}

The authors are grateful to the UK Engineering and Physical Sciences Research Council for financial support of this work. Dr Graham Eaton is also acknowledged for recording the low energy EI mass spectrum of gaseous 1-pentanol.

\section{References}

1 A. J. Stace, Chem. Phys. Lett., 1983, 99, 470.

2 A. J. Stace, J. Am. Chem. Soc., 1984, 106, 4380.

3 A. J. Stace, J. Phys. Chem., 1983, 87, 2286.

4 A. J. Stace, J. Am. Chem. Soc., 1985, 107, 755.

5 A. J. Stace, J. Phys. Chem., 1987, 91, 1509.

6 D. M. Bernard, N. G. Gotts and A. J. Stace, Int. J. Mass Spectrom. Ion Processes, 1990, 95, 327.

7 J. P. Toennies and A. F. Vilesov, Annu. Rev. Phys. Chem., 1998, 49, 1.

8 J. P. Toennies and A. F. Vilesov, Angew. Chem., Int. Ed., 2004, 43, 2622.

9 T. Jiang and J. A. Northby, Phys. Rev. Lett., 1992, 68, 2620.

10 A. Scheidemann, B. Schilling and J. P. Toennies, J. Phys. Chem., 1993, 97, 2128

11 M. Lewerenz, B. Schilling and J. P. Toennies, Chem. Phys. Lett., 1993, 206, 381.

12 B. E. Callicoatt, D. D. Mar, V. A. Apkarian and K. C. Janda, J. Chem. Phys., 1996, 105, 7872.

13 B. E. Callicoatt, K. Förde, L. F. Jung, T. Ruchti and K. C. Janda, J. Chem. Phys., 1996, 109, 10195.

14 R. Frochtenicht, U. Henn, J. P. Toennies, A. Ding, M. FieberErdmann and T. Drewello, J. Chem. Phys., 1996, 104, 2548.

15 B. E. Callicoatt, K. Förde, T. Ruchti, L. Jung, K. C. Janda and N. Halberstadt, J. Chem. Phys., 1998, 108, 9371.

16 T. Ruchti, K. Förde, B. E. Callicoatt, H. Ludwigs and K. C. Janda, J. Chem. Phys., 1998, 109, 10679.

17 W. K. Lewis, B. E. Applegate, J. Sztáray, B. Sztáray, T. Baer, R. J. Bemish and R. E. Miller, J. Am. Chem. Soc., 2004, 126, 11283.

18 M. N. Slipchenko, S. Kuma, T. Momose and A. F. Vilesov, Rev. Sci. Instrum., 2002, 73, 3600.

19 S. Yang, S. M. Brereton and A. M. Ellis, Rev. Sci. Instrum. DOI: $10.1063 / 1.2093766$

20 NIST Mass Spec Data Center, S. E. Stein, director, in NIST Chemistry WebBook, NIST Standard Reference Database Number 69, ed. P. J. Linstrom and W. G. Mallard, National Institute of Standards and Technology, Gaithersburg, MD, 2001, http://webbook.nist.gov/.

21 H. Budzikiewicz, C. Djerassi and D. H. Williams, Mass Spectrometry of Organic Compounds, Holden-Day Inc., San Francisco, CA, 1967.

22 F. W. McLafferty and F. Tureček, Interpretation of Mass Spectra, University Science Books, Mill Valley, CA, 1993.

23 G. D. Stein, Surf. Sci., 1985, 156, 44.

24 Y. Niwa, T. Nishimura and T. Tsuchiya, Int. J. Mass Spectrom. Ion Phys., 1982, 42, 91.

25 R. D. Bowen and A. Maccoll, Org. Mass Spectrom., 1984, 19, 379.

26 A. V. Kanaev, L. Museur, T. Laarmann, S. Monticone, M. C. Castex, K. von Haeften and T. Möller, J. Chem. Phys., 2001, 115, 10248.

27 A. Nakayama and K. Yamashita, J. Chem. Phys., 2000, 112, 10966.

28 S. Yang, S. M. Brereton, M. D. Wheeler and A. M. Ellis, in preparation. 